\title{
Quantification of antibiotic use on dairy farms in Pennsylvania
}

\author{
Laurel E. Redding, ${ }^{*}$ Joseph Bender, and Linda Baker \\ Department of Clinical Studies, School of Veterinary Medicine, University of Pennsylvania, Kennett Square 19348
}

\section{ABSTRACT}

Antibiotic use data are critical for drawing conclusions about the epidemiological connections between antibiotic use in farms animals, antibiotic resistance, animal health, and human health. The goal of this study was to quantitatively and qualitatively characterize antibiotic use on dairy farms in Pennsylvania, the state with second largest number of dairy farms nationally. A survey was sent to $10 \%$ of the 6,580 dairy farms registered in Pennsylvania and completed by 235 producers (response rate of $36 \%$ ). Data on antibiotic use in the previous month and in the previous 6 mo were collected based on farmer self-report, using either recall or treatment records. Two metrics were used to quantify antibiotic consumption: animal-defined daily doses (ADD) and days of therapy (DOT), a metric used in human medicine for purposes of antimicrobial stewardship. Across all farms, 24,444 ADD and 19,029 DOT were reported, representing treatment incidences of 4.2 ADD/1,000 animal-days and $3.3 \mathrm{DOT} / 1,000$ animal-days. These rates were generally lower than those found in other states and countries. The main indication for antibiotic use was mastitis, and firstgeneration cephalosporins were the most commonly used class of antibiotic for all indications, followed by penicillins and third-generation cephalosporins. Trends in use were similar for ADD and DOT, but the numbers of recorded DOT and associated treatment incidences were generally lower than the number of ADD and associated treatment incidences. Rates of treatment were significantly associated with herd size. This study is the first to quantify antibiotic use on dairy farms in Pennsylvania and the first to use the DOT metric in a dairy setting.

Key words: dairy cow, antibiotic, antimicrobial, dairy farm

Received June 16, 2018

Accepted October 18, 2018.

*Corresponding author: lredding@vet.upenn.edu

\section{INTRODUCTION}

Antimicrobial use data are critical for understanding how antimicrobials are used on farms and how use may have changed over time. These data are also needed to understand the relationship between antimicrobial use and antimicrobial resistance and to promote the judicious use of antimicrobials. On dairy farms, antimicrobials are used for a variety of therapeutic (e.g., mastitis, metritis, respiratory disease, foot disease) and prophylactic (e.g., dry-cow therapy, medicated milk replacer in calves) purposes, and many of the antibiotics that are commonly used in dairy production are considered critically important by the World Health Organization (WHO, 2013a).

Several studies have described antibiotic use on dairy farms in the United States (Zwald et al., 2004; Sawant et al., 2005; Raymond et al., 2006; Pol and Ruegg, 2007). These studies mostly describe the proportion of farms using antimicrobial treatments in a predefined period and the ranking of drug types by active ingredients, with most studies finding considerable variation in patterns of use across farms. However, very little information is available on the quantities of antibiotics used on dairy farms in the United States: only one study in Wisconsin attempted to quantify antimicrobial use using the animal-defined daily dose (ADD) and treatment incidence (ADD/cow-year) metrics (Pol and Ruegg, 2007). Quantitative antimicrobial use data are critical for comparing (e.g., by farm, region, country) and benchmarking use. However, quantifying use is made difficult by the variety and the lack of standardized metrics. For example, in Canada and Europe, studies quantifying antibiotic use on dairy farms have reported on the percentage of farms using a product or animals treated with that product (Brunton et al., 2012; Stevens et al., 2016), the dose of drug ( $\mathrm{mg} / \mathrm{kg}$ of biomass; González et al., 2010; Obritzhauser et al., 2016; Bryan and Hea, 2017), the number of ADD (Pol and Ruegg, 2007; Saini et al., 2012; Stevens et al., 2016), and the number of prescribed daily doses (Obritzhauser et al., 2016). The variety of ways that antibiotic use can be quantified makes the interpretation of antibiotic use data difficult for relevant stakeholders (e.g., producers, veterinarians, 
public health officials), and different stakeholders may be interested in different metrics. For example, the total volume of a drug used on a farm might be useful for a policymaker, whereas the number of ADD per cow per year may be more useful for a producer or a veterinarian. Moreover, as was elegantly demonstrated by Mills et al. (2018), the use of different metrics can result in different rankings of use by farm. Given the current lack of a "gold standard" metric, it is our opinion that antimicrobial use data are best presented using a variety of metrics. To that end, the goal of this study was to quantify antibiotic use on Pennsylvania dairy farms - where use has been characterized (Sawant et al., 2005) but never quantified - using different metrics and to assess the association between certain herd-level factors and antibiotic use. Specifically, we used the proportion of farms, number of ADD, and the number of days of therapy (DOT), a novel metric used in human medicine representing the total number of days that patients received antibiotic treatments during a defined study period. With increasing interest in antimicrobial stewardship in veterinary medicine, it is critical to have baseline antibiotic use data that can be used to evaluate changes in antibiotic use patterns associated with interventions (such as the Veterinary Feed Directive; FDA, 2009), changing consumer patterns, and management practices.

\section{MATERIALS AND METHODS}

\section{Survey Instrument}

A survey instrument was developed based on previously published surveys of antimicrobial use on dairy farms (Zwald et al., 2004; Sawant et al., 2005; Raymond et al., 2006; Saini et al., 2012; Léger et al., 2017) and discussions among the research team. A paper form was created and an online form of the survey was developed using the REDCap electronic data capture tool hosted at the University of Pennsylvania (Harris et al., 2009). Both forms of the survey were piloted on 3 dairy farms to clarify any ambiguous questions and refine the content of the survey. The survey was designed to collect information on the herd, management factors relating to antibiotic use (e.g., use of treatment protocols, record keeping), calf feeding, the number of animals experiencing various diseases in the preceding 1 and $6 \mathrm{mo}$, the type of antibiotics used to treat these diseases, and the dose, frequency, and duration of treatment using either recall or treatment records if they existed (Supplemental File S1; https://doi.org/10.3168/jds.2018-15224). A visual aid consisting of photos of the packages and labels for most antibiotics used in dairy practice was included in the survey, as this type of visual aid has been shown to enhance recall of drug use (Kimmel et al., 2003). The survey was sent out in November 2017, and farmers were asked to return or complete the survey within 3 mo. The study was deemed exempt from requiring human subjects approval by the Institutional Review Board of the University of Pennsylvania.

\section{Study Population}

A list of the 6,580 registered dairy farmers in Pennsylvania was provided by the Center for Dairy Excellence (Harrisburg, PA), and a random number generator was used to randomly select $10 \%$ of the farms $(658$ farms) for participation. A package containing a cover letter, a paper survey, a postage-paid return addressed envelope, and a $\$ 5$ cash incentive was sent to each of these 658 dairy farmers. The cover letter contained a description of the study and a link to the online form with instructions to either fill out and return the paper survey or fill out the online form.

\section{Data Analysis}

Drug use data from the paper surveys were entered into a REDCap database, and all data were exported to and analyzed in Stata (Stata Corp., College Station, TX). All surveys were manually checked to detect errors in data entry.

Antibiotic drug use data were quantified using a variety of metrics. For each antibiotic class (first- and third-generation cephalosporins, penicillins, tetracyclines, sulfonamides, macrolides, fluoroquinolones, and phenicols), the total amount of active ingredient (milligrams) was calculated as the number of units (milliliters, tablets) multiplied by the concentration of the drug, the daily frequency of use, the number of days of treatment, and the number of animals treated, as follows:

Active substance $(\mathrm{mg})=$ no. of animals treated in study period $\times$ drug concentration $(\mathrm{mg} / \mathrm{mL})$ $\times$ units per treatment $(\mathrm{mL}) \times$ treatments per day $\times$ days of treatment.

The animal-defined daily dose $\left(\mathrm{ADD}_{\text {vet }}\right.$, in $\left.\mathrm{mg} / \mathrm{d}\right)$ was the assumed average daily dose for an animal and was calculated as the average labeled dose (defined daily dose, $\mathbf{D D D}_{\text {vet }}$, in $\mathrm{mg} / \mathrm{kg}$ ) multiplied by the standard weight of an animal (50 kg for calves, $200 \mathrm{~kg}$ for heifers, $600 \mathrm{~kg}$ for an adult cow; Jensen et al., 2004). If 
a single dose was listed on the label, that dose was the $\mathrm{DDD}_{\text {vet. }}$. If a range of doses was given, the mean dose was used as the $\mathrm{DDD}_{\text {vet }}$ (Saini et al., 2012). For combination products (e.g., penicillin-streptomycin), the $\mathrm{DDD}_{\text {vet }}$ was estimated for the main substance, in accordance with the guidelines for DDD assignment in human medicine (World Health Organization, 2013b). The $\mathrm{DDD}_{\text {vet }}$ for intramammary products was assigned according to guidelines of the European Medicines Agency (European Medicines Agency, 2015), with 4 intramammary applicators representing $1 \mathrm{DDD}_{\text {vet }}$ for dry-cow therapy (DCT), and 1 applicator representing $1 \mathrm{DDD}_{\text {vet }}$ for lactating-cow therapy. The total number of animals on the farm was assumed to vary minimally (Saini et al., 2012) over the course of 6 mo and was therefore treated as constant.

The number of ADD used in the previous 1 and 6 mo was calculated by dividing the total amount of active ingredient (mg) used during that period by the $\mathrm{ADD}_{\text {vet }}$ $(\mathrm{mg} / \mathrm{d})$ :

$$
\mathrm{ADD}=\frac{\mathrm{mg} \text { of active ingredient }}{\mathrm{ADD}_{\mathrm{vet}}}
$$

A treatment incidence per 1,000 animal-days $\left(\mathrm{TI}_{\mathrm{ADD}}\right)$ was then calculated:

$$
\mathrm{TI}_{\mathrm{ADD}}=\frac{\mathrm{ADD}}{\text { animals at risk } \times \text { days at risk }} \times 1,000 .
$$

Days of antibiotic therapy is a metric used in human medicine that is now preferred over the daily defined dose by the Infectious Disease Society of America (Barlam et al., 2016). For each class of antibiotic, DOT was calculated as the product of the number of animals treated with that drug class and the duration of therapy:

DOT $=$ no. of animals treated $\times$ duration of therapy.

A treatment incidence per 1,000 animal-days $\left(\mathrm{TI}_{\mathrm{DOT}}\right)$ was then calculated:

$$
\mathrm{TI}_{\mathrm{DOT}}=\frac{\mathrm{DOT}}{\text { animals at risk } \times \text { days at risk }} \times 1,000 .
$$

Differences in treatment incidences (for both ADD and DOT) by herd size and management factors were assessed using ANOVA or the Kruskal-Wallis test, as appropriate. Correlations between the different metrics were assessed using the Spearman rank correlation test.

\section{RESULTS}

\section{Survey Responses}

Of the 658 surveys sent out, 64 were returned not completed: 31 were returned by the postal service unopened, 23 producers had sold their herds, and 10 returned the survey without providing any data. Three producers filled out the survey online, and 232 producers returned completed paper surveys, for a total of 235 (response rate of $35.7 \%$ ). The 235 respondents represented 137 Zip Codes throughout Pennsylvania (Figure 1) and accounted for 15,974 lactating cows, 2,445 dry cows, 10,535 heifers, 3,628 calves, and 286 bulls, or 32,868 dairy animals.

\section{Farm Demographic Data}

Using the herd size categorization of the National Animal Health Monitoring System (NAHMS; USDAAPHIS-NAHMS, 2016), 20 (9\%) were very small $(<30$ cows), 162 (70\%) were small (30-99 cows), 50 (21\%) were medium (100-499 cows), and 2 (1\%) were very large (>500 cows). The mean number of lactating cows on surveyed farms (65) was close to the state average of 70 (Center for Dairy Excellence, 2018). Demographic data on the farms are provided in Table 1. Sixteen farms were certified organic, and 3 of those (19\%) reported having used antibiotics during the previous 6 mo. Sixty percent of farms had written treatment records and $63 \%$ followed antibiotic use protocols developed by their veterinarians. Almost all of the farms (93\%) raised their calves on site.

\section{Disease Frequencies}

The frequencies of selected diseases are reported in Table 2. Mastitis was the most commonly occurring disease, followed by calf diarrhea and calf respiratory disease. During the previous 1 and 6 mo, 25 and $18 \%$ of farms, respectively, reported experiencing no cases of treated disease on their farms. The percentage of animals on the farm affected tended to be low (median of 0 for most disease categories). Twelve percent of all animals across all farms experienced at least one treated disease event during the 6-mo period.

\section{Nontherapeutic Use of Antibiotics}

Calves. Medicated milk replacers in the United States can contain tetracycline, neomycin, or coccidiostats such as monensin, lasalocid, or decoquinate. Forty-one (18.6\%) farms used medicated milk replacer, 
Table 1. Demographic data from 235 dairy farms in Pennsylvania that participated in a survey of antibiotic use in 2017

\begin{tabular}{|c|c|c|c|}
\hline Item & Median & Range & $\begin{array}{c}\text { Total across } \\
\text { all farms }\end{array}$ \\
\hline \multicolumn{4}{|l|}{ Animal factors } \\
\hline Lactating cows & 50 & $0-600$ & 15,974 \\
\hline Dry cows & 8 & $0-75$ & 2,445 \\
\hline Heifers & 30 & $0-575$ & 10,535 \\
\hline Preweaned calves & 10 & $0-110$ & 3,628 \\
\hline Bulls & 0 & $0-30$ & 286 \\
\hline Total & 101 & $0-1,305$ & 32,868 \\
\hline \multicolumn{4}{|c|}{ Farm factors, number $(\%), \mathrm{n}=235$} \\
\hline \multicolumn{3}{|c|}{ Organic } & $15(6.4)$ \\
\hline \multicolumn{3}{|c|}{ Calves raised on site } & $202(87)$ \\
\hline \multicolumn{3}{|c|}{ The farm maintains written treatment records } & $140(60)$ \\
\hline \multicolumn{3}{|c|}{ The farm has a protocol from veterinarian for administering antibiotics } & $142(63)$ \\
\hline \multicolumn{4}{|c|}{ How often farmers who have protocols from veterinarians follow them } \\
\hline \multicolumn{3}{|c|}{ Never } & $2(1.4)$ \\
\hline \multicolumn{3}{|l|}{ Sometimes } & $55(39)$ \\
\hline \multicolumn{3}{|c|}{ Always } & $90(63)$ \\
\hline \multicolumn{4}{|c|}{ Cows treated with dry-cow therapy } \\
\hline \multicolumn{3}{|c|}{$0 \%$} & $32(14)$ \\
\hline \multicolumn{3}{|l|}{$0-49 \%$} & $19(8)$ \\
\hline \multicolumn{3}{|l|}{$50-99 \%$} & $35(15)$ \\
\hline \multicolumn{3}{|l|}{$100 \%$} & $137(58)$ \\
\hline
\end{tabular}

but only $7(3 \%)$ reported using a milk replacer with an antibiotic ( 1 with chlortetracycline, 6 with neomycin + oxytetracycline). The farms that fed the milk replacer with neomycin + oxytetracycline mostly fed their calves $2 \mathrm{~L} / \mathrm{d}$, representing $4 \mathrm{mg}$ of tetracycline and $50 \mathrm{mg}$ of neomycin per calf per day. The use of medicated milk replacer was not associated with either of the treatment rates $(\mathrm{ADD} / 1,000$ animal-days, $P=0.61 ; \mathrm{DOT} / 1,000$ animal-days, $P=0.89$ ).

Dry-Cow Therapy. A total of $85 \%$ of farms used DCT: $61 \%$ treated all of their cows, $16 \%$ treated 50 to $99 \%$ of cows, and $8 \%$ treated less than $50 \%$ of their cows. Across all farms, DCT was administered to 5,941 cows in the previous 6 mo. First-generation
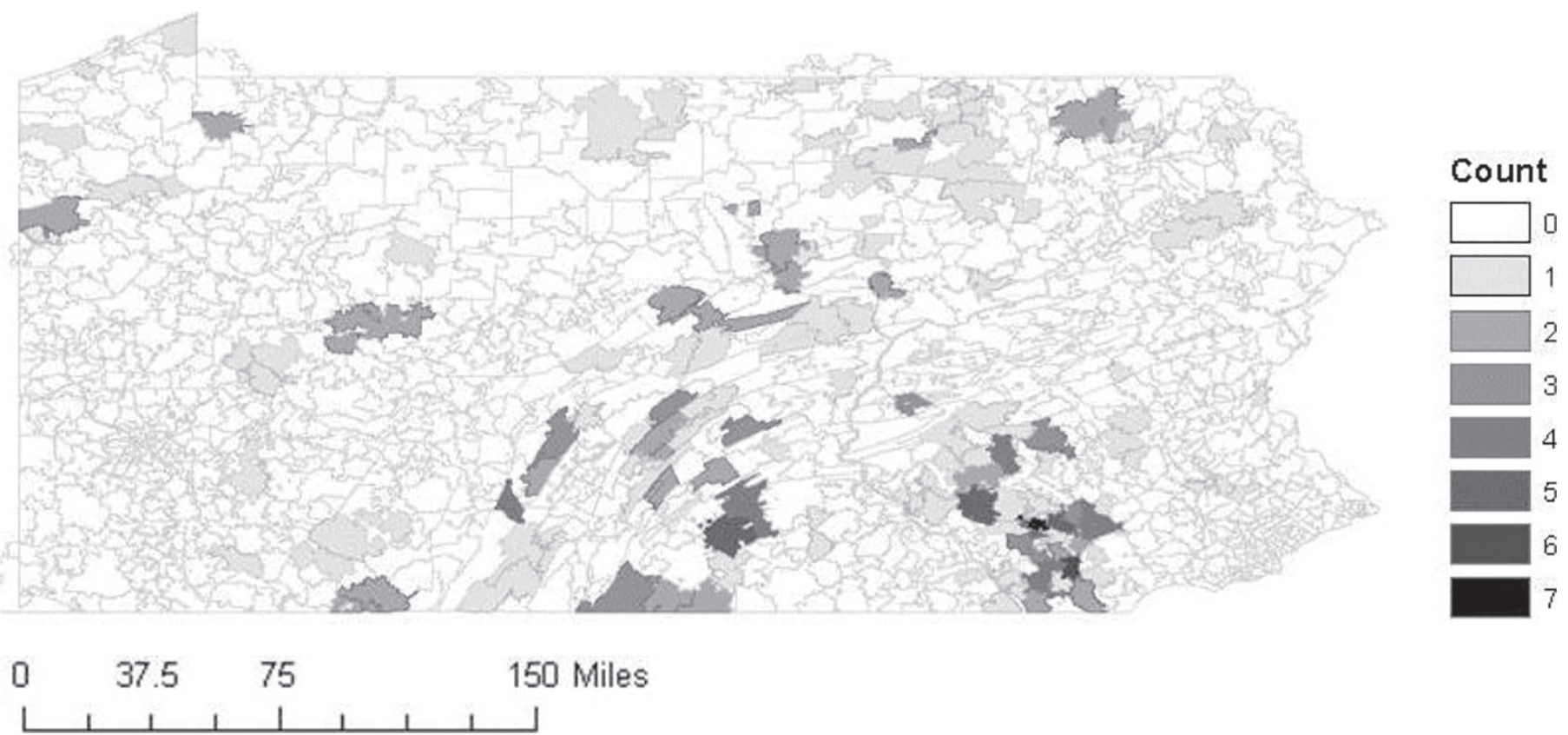

Figure 1. Counts of survey responses by postal code for a survey on antibiotic use completed by 235 dairy farmers from Pennsylvania in 2017 . 
Table 2. Frequency of selected diseases on 235 dairy farms in Pennsylvania that participated in a survey of antibiotic use in 2017

\begin{tabular}{|c|c|c|c|c|c|c|}
\hline Disease & \multicolumn{2}{|c|}{$\begin{array}{l}\text { No. }(\%) \text { of farms with } \\
\text { reported episodes of } \\
\text { treated disease }\end{array}$} & \multicolumn{2}{|c|}{$\begin{array}{l}\text { No. of treated episodes } \\
\text { of disease ( } \% \text { of animals) } \\
\text { across all farms }\end{array}$} & \multicolumn{2}{|c|}{$\begin{array}{c}\text { Median (range) percentage } \\
\text { of treated animals } \\
\text { at farm level }\end{array}$} \\
\hline Calf diarrhea & $70(30)$ & $113(49)$ & $245 / 3,628(6.7)$ & $794 / 3628(22)$ & $0(0-42.9)$ & $5.72(0-100)$ \\
\hline Calf respiratory disease & $83(36)$ & $97(42)$ & $289 / 3,628(8.0)$ & $533 / 3628(15)$ & $0(0-100)$ & $0(0-100)$ \\
\hline Respiratory disease & $15(6)$ & $45(19)$ & $40 / 32,868(0.12)$ & $116 / 32,868(0.40)$ & $0(0-1.54)$ & $0(0-3.9)$ \\
\hline Foot disease & $71(31)$ & $90(39)$ & $202 / 32,868(0.61)$ & $599 / 32,868(2.1)$ & $0(0-9.61)$ & $0(0-33)$ \\
\hline $\begin{array}{l}\text { Other disease (e.g., hardware } \\
\text { disease, pink eye, injury) }\end{array}$ & $28(12)$ & $35(14)$ & $42 / 32,868(0.13)$ & $125 / 32,868(0.41)$ & $0(0-1.92)$ & $0(0-5.3)$ \\
\hline Total (all diseases) & $176(75)$ & $193(82)$ & $1,375 / 32,868(4.2)$ & $3,872 / 32,868(12)$ & $2.5(0-34)$ & $8(0-84)$ \\
\hline
\end{tabular}

cephalosporins (e.g., cephapirin) were used most often (664 ADD in previous 1 mo; 3,740 ADD in previous 6 mo), followed by penicillins (e.g., procaine penicillin $\mathrm{G}$, cloxacillin) and third-generation cephalosporins (e.g., ceftiofur). The median (range) treatment incidence for DCT was 1.9 (0-5.4) ADD/1,000 animal-days.

\section{Therapeutic Use of Antibiotics}

Eighty-two percent of farms used antibiotics at some point during the previous 6-mo period, and the rates of use for all antibiotics across all farms were 4.2 ADD/1,000 animal-days and 3.3 DOT/1,000 animaldays. A breakdown of antibiotic classes by disease is shown in Figure 2. Antibiotic use data were highly right-skewed (Figure 3); therefore, median levels and ranges of use are presented by drug class in Tables 3 and 4 . The variability in treatment incidence was relatively low across all farms, with an interquartile range of 1.7 to $3.7 \mathrm{ADD} / 1,000$ animal-days and 0.7 to 3.2 DOT/1,000 animal-days. Antibiotic use data by drug class are represented visually in Figures 3, 4, and 5, with similar trends in ranking of drug class for all metrics. First-generation cephalosporins were used on the greatest number of farms (58\%) and were associated with the highest treatment incidence $(2.3 \mathrm{ADD} / 1,000$ animal-days and 1.7 DOT/1,000 animal-days), whereas sulfonamides were used on the fewest farms (4\%) and associated with the lowest treatment incidence $(0.1$ ADD/1,000 animal-days; Table 3). The greatest number of ADD was used to treat mastitis $(8,664)$, and the lowest (284) to treat other diseases (e.g., hardware disease, injury).

Similar patterns were seen for DOT as for ADD (Table 4). The greatest number of DOT was associated with treatment of mastitis $(8,226 \mathrm{DOT})$, and the fewest for other diseases (284). The overall treatment incidences were similar for ADD and DOT (Figure 6).
The absolute number of DOT tended to be lower than the number of ADD for all antibiotic classes except macrolides, phenicols, and sulfonamides, although the total number of ADD and DOT were highly correlated (Spearman rank correlation $=0.85 ; P<0.001$ ). A similar situation was found with the treatment incidences (Spearman rank correlation $=0.81 ; P<0.001$ ). Across all farms and all disease categories, there were 19,029 DOT. The treatment incidences differed significantly by farm size (Spearman rank correlation coefficient $=0.2, P<0.001$; Table 5), with larger farms having greater treatment incidences than smaller farms. No off-label use by drug class was noted.

\section{DISCUSSION}

To our knowledge, this is the first study to quantify antibiotic use on Pennsylvania dairy farms. Pennsylvania is ranked sixth in total milk production nationally and has the second largest number of dairy farms after Wisconsin. The last study to describe antibiotic use on dairy farms in Pennsylvania was produced by Sawant et al. (2005) using data collected in 2001-2002. Since then, the number of dairy farms in Pennsylvania has declined significantly, although total milk production and cows per farm have increased (Center for Dairy Excellence, 2018). Management, drug availability, consumer trends, and regulatory drivers of drug use (e.g., the Veterinary Feed Directive) have changed greatly since 2002, resulting in the need to update the current literature regarding antibiotic drug use.

The overall treatment rates for all antibiotics across all farms were $4.2 \mathrm{ADD} / 1,000$ animal-days and 3.3 DOT/1,000 animal-days, which is equivalent to 1.5 $\mathrm{ADD} /$ animal-year and 1.2 DOT/animal-year. The variability in treatment incidences (i.e., $\mathrm{ADD} / 1,000$ animaldays and DOT/1,000 animal-days) was relatively low, suggesting that animals were exposed to antibiotics at 
a)

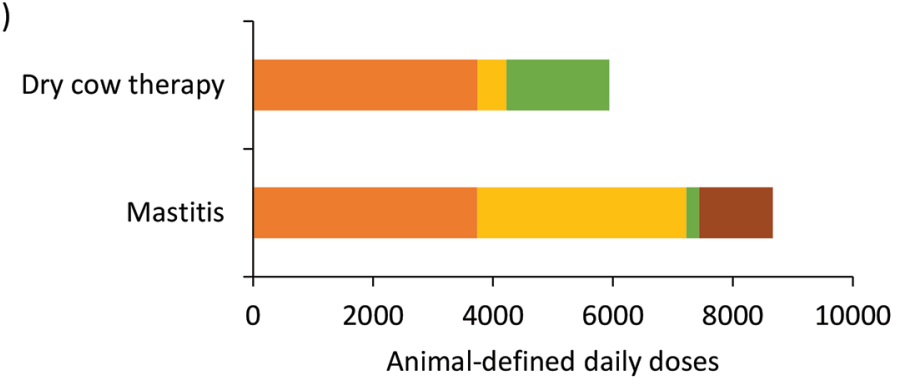

b)

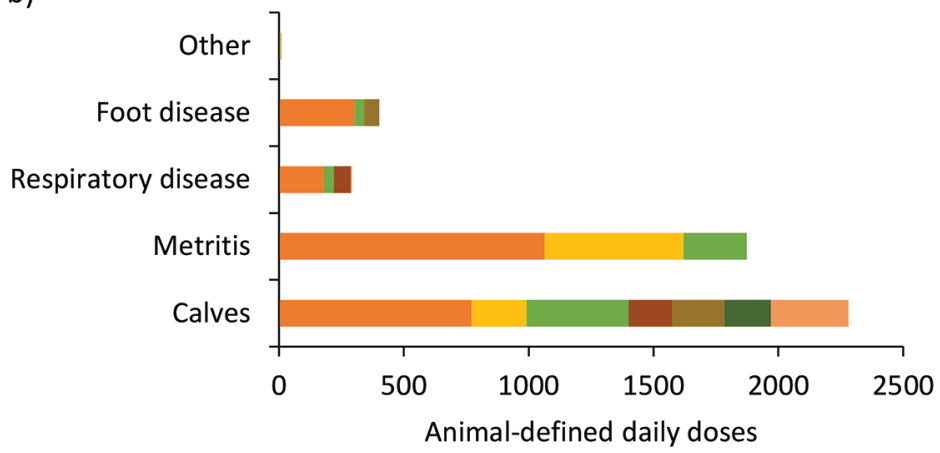

1st generation cephalosporin

3rd generation cephalosporins

Penicillins

- Lincosamides

Figure 2. Breakdown of animal-defined daily doses (ADD) of antibiotics used in the previous 6 mo on 235 dairy herds in Pennsylvania in 2017: (a) antibiotics used for dry-cow therapy and mastitis by antibiotic class and (b) antibiotics used for other diseases by antibiotic class (note difference in scale for ADD relative to that of panel a).

similar rates across most farms. We did see a statistically significant difference in treatment incidence by farm size, but the absolute difference in incidence was relatively small: on small and medium-sized farms, which accounted for $91 \%$ of the farms in our study, the difference in the median treatment incidence between these types of farms was only 0.6 ADD/1,000 animaldays.

Few studies have quantified antibiotic use on dairy farms in the United States. Pol and Ruegg (2007) described antibiotic use on dairy farms in Wisconsin and reported a treatment incidence of 5.43 defined daily doses (i.e., ADD) per cow per year, which would be equivalent to 14.8 ADD/1,000 cow-days. In Canada, Saini et al. (2012) reported an overall treatment incidence (or antibiotic drug use rate) of 14.35 ADD/1,000 cow-days. In other countries, overall treatment incidences have ranged from 1.3 ADD/cow per year (or 3.56 ADD/1,000 cow-days) in Austria, (Obritzhauser et al., 2016) to $3.24 \mathrm{ADD} /$ cow per year (or $8.87 \mathrm{ADD} / 1,000$ cow-days) in the United Kingdom (Hyde et al., 2017) and 20.78 ADD/1,000 cow-days in Belgium (Stevens et al., 2016).

Several factors might explain the relatively lower rates of treatment in this study. Pennsylvania dairy farms might simply use fewer antibiotics, either due to lower disease rates or different management practices.
For example, less frequent use of medicated milk replacers or a transition toward targeted rather than blanket DCT could reduce overall use. Pennsylvania also has many Amish and Mennonite farmers, who have been found to use antibiotics less frequently (Schewe and Brock, 2018). Although we did not ascertain whether respondents identified as Amish or Mennonite, given the large numbers of such families in Pennsylvania, it is highly likely they were represented among survey respondents. At the time of the survey, because milk prices were low, producers might also have been more reluctant to treat sick cows rather than cull them, a finding that we have observed anecdotally. We found that treatment incidence was significantly associated with herd size, and the farms surveyed here tended to be smaller than the farms surveyed in other studies.

The lower rates may also have been associated with poor recall of farmers. Forty percent of farmers in the study did not have written treatment records and may have not recalled all instances of treatment. Moreover, farmers were asked to recall units of the drug given (i.e., milliliters of injectable drug), which may have resulted in inaccuracies. However, in our experience, dairy farmers tend to follow protocols with standardized doses developed by their veterinarian, and $63 \%$ of the surveyed farms reported following such protocols. We believe that the standardization of dosing through 


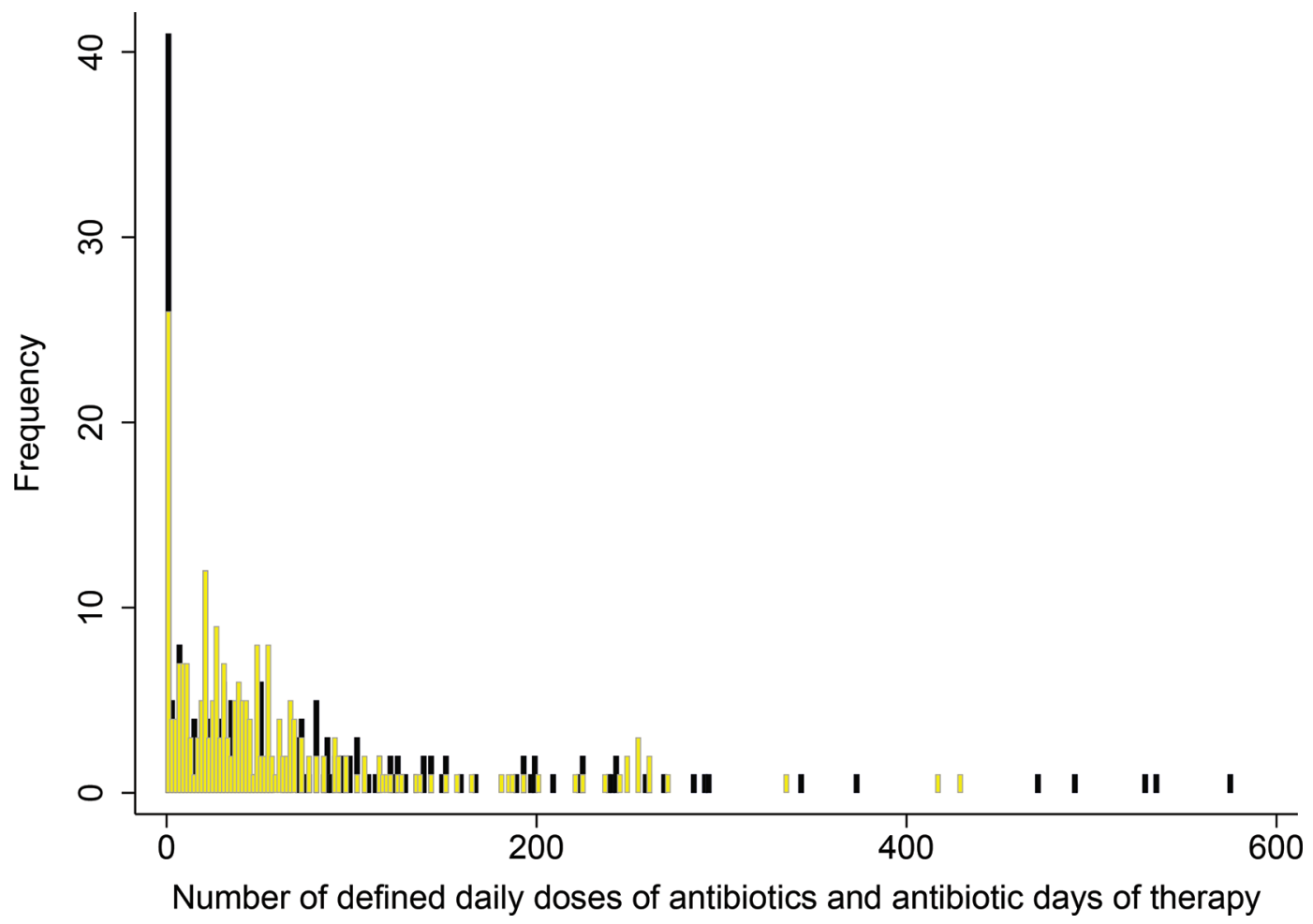

Figure 3. Distribution of the number of animal-defined daily doses (ADD; black boxes) and days of antibiotic therapy (DOT; yellow boxes) used in the previous 6 mo on 235 dairy farms in Pennsylvania in 2017.

protocols - and the resultant repeated use of the same doses and volumes - enhances recall of units used.

Finally, the lower rates may also be related to how the treatment incidences were calculated. Standard weights are used in the calculations (here, $600 \mathrm{~kg}$ for an adult cow, $200 \mathrm{~kg}$ for a heifer, and $50 \mathrm{~kg}$ for a calf; Jensen et al., 2004); however, if these standard weights are not representative of the weights of animals on the farm, the calculated number of ADD used can be inaccurate. The number of animals at risk used to calculate the treatment incidence could also vary. For example, studies using garbage can audit systems, such as that of Saini et al. (2012), collect discarded packaging used for all animals (e.g., calves, heifers, and adult cows),

Table 3. Animal defined daily doses (ADD) used on 235 dairy farms in Pennsylvania in 2017

\begin{tabular}{|c|c|c|c|c|c|c|c|}
\hline \multirow[b]{2}{*}{ Antimicrobial class } & \multirow{2}{*}{$\begin{array}{l}\text { No. of herds } \\
(\%) \text { using } \\
\text { drug any time } \\
\text { during study }\end{array}$} & \multicolumn{2}{|c|}{$\begin{array}{c}\text { ADD used } \\
\text { in previous } 1 \mathrm{mo}\end{array}$} & \multicolumn{2}{|c|}{$\begin{array}{c}\text { ADD used } \\
\text { in previous } 6 \text { mo }\end{array}$} & \multicolumn{2}{|c|}{$\begin{array}{c}\text { Treatment incidence } \\
\text { (ADD/1,000 animal-days) }\end{array}$} \\
\hline & & $\begin{array}{l}\text { Median } \\
\text { (range) } \\
\text { per farm }\end{array}$ & $\begin{array}{c}\text { Total } \\
\text { across } \\
\text { all farms }\end{array}$ & $\begin{array}{l}\text { Median } \\
\text { (range) } \\
\text { per farm }\end{array}$ & $\begin{array}{c}\text { Total } \\
\text { across } \\
\text { all farms }\end{array}$ & $\begin{array}{l}\text { Median } \\
\text { (range) }\end{array}$ & $\begin{array}{c}\text { Total } \\
\text { across } \\
\text { all farms }\end{array}$ \\
\hline $\begin{array}{l}\text { Cephalosporins, first } \\
\text { generation }\end{array}$ & $134(58)$ & $6.0(0-126)$ & 1,363 & $30(2-255)$ & 7,619 & $0.24(0-27.9)$ & 2.3 \\
\hline Tetracyclines & $49(21)$ & $0.5(0-31.6)$ & 117 & $4.5(0-303)$ & 2,642 & $0.15(0-27.2)$ & 0.45 \\
\hline Lincosamides & $30(12)$ & $6(0-36)$ & 264 & $36(3-171)$ & 1,286 & $2.5(0.32-15.1)$ & 0.39 \\
\hline Macrolides & $25(11)$ & $0.19(0-33.5)$ & 171 & $0.62(0.01-96)$ & 242 & $0.015(0-4.8)$ & 0.24 \\
\hline Fluoroquinolones & $19(8.1)$ & $1.2(0.2-24)$ & 89.9 & $5.0(0.2-72)$ & 197 & $0.11(0.008-2.2)$ & 0.20 \\
\hline Phenicols & $15(6.4)$ & $3.6(0-35.2)$ & 116 & $7.2(1.1-131)$ & 316 & $0.19(0.04-3.7)$ & 0.52 \\
\hline $\begin{array}{l}\text { Sulfonamides } \\
(+ \text { trimethoprim })\end{array}$ & $10(4)$ & $0.5(0-31.6)$ & 117 & $4.5(0.0005-27)$ & 270 & $0.47(0.02-3.0)$ & 0.10 \\
\hline
\end{tabular}


Table 4. Days of antibiotic therapy (DOT) used on 235 dairy farms in Pennsylvania in 2017

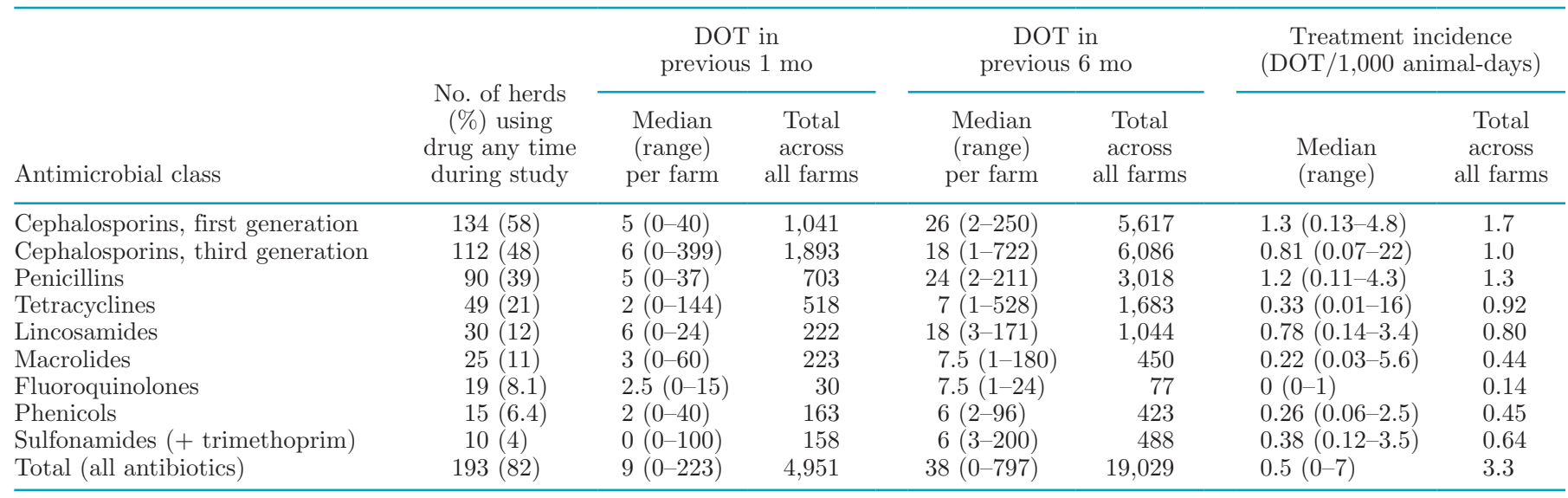

but the denominator for treatment incidence included only adult cows, thus inflating the calculated treatment rate. Finally, a mean labeled dose is used to calculate the number of ADD used, and if producers used a lower dose than the mean, the treatment incidence will appear lower.

The DOT metric has not been extensively used in the veterinary setting or specifically at the dairy herd level. In human medicine, DOT is now preferred over the DDD by the Infectious Disease Society of America for purposes of antimicrobial stewardship (Barlam et al., 2016). This metric is conceptually easier to understand than the DDD or ADD and easier to calculate. However, it is useful only to the extent that there are accepted targets against which to benchmark. At this stage, there are no such targets for DOT on dairy farms. This study represents a preliminary step in articulating baseline levels on Pennsylvania dairy farms. In theory, if the dose used by the producer is the same as the $\mathrm{DDD}_{\text {vet }}$, then the DOT should approximate the $\mathrm{ADD}$. If the DOT is lower than the ADD, as was often the case in this study (see Figure 3), then either the dose used by farmer was greater than the $\mathrm{DDD}_{\text {vet }}$ or the standard weight of the animal was lower than the actual estimated weight of the treated animal. In this study, we used a standard weight of $600 \mathrm{~kg}$ for an adult cow. In other studies, standard weights of $425 \mathrm{~kg}$ (Hyde et al., 2017), $500 \mathrm{~kg}$ (Obritzhauser et al., 2016), $600 \mathrm{~kg}$ (Saini et al., 2012; Stevens et al., 2016), and $680 \mathrm{~kg}$ (Pol and Ruegg, 2007) have been used.

The patterns of drug use and disease occurrence found in our study were similar to those found in other studies. Most studies have found mastitis to be the primary indication for antibiotics (Pol and Ruegg, 2007; Kuipers et al., 2016; Stevens et al., 2016; Hyde et al., 2017 ); in our study, mastitis occurred on $63 \%$ of farms and was associated with the use of 8,664 ADD dur- ing the study period. This finding, although consistent with other studies, may also be related to the fact that course-based metrics (e.g., ADD and DOT/1,000 animal-days) were used, rather than mass-based metrics (e.g., mg $/ \mathrm{kg}$ ). If a mass-based metric had been used, a different ranking of use by indication may have been found, because intramammary products contain small quantities of antibiotics. Interestingly, in Pennsylvania, Sawant et al. (2005) found calf enteritis (i.e., diarrhea) to be the single most commonly treated disease, occurring in $36 \%$ of the calf population. In our study, $49 \%$ of farms treated calves for enteritis in the previous 6 mo, accounting for 2,336 d of antibiotic therapy; however, more antibiotics were used for mastitis than for calf diarrhea. In contrast to other studies, we did not determine the percent of disease episodes that were treated with antibiotics. In Pennsylvania, this figure was found to range from 1 to $36 \%$ (Sawant et al., 2005); therefore, it is likely that the surveyed farms experienced additional episodes of disease that were not treated with antibiotics.

The ranking of antibiotics differed by study but in general, $\beta$-lactams (e.g., penicillins and cephalosporins) accounted for the greatest use, as was reported by Sawant et al. (2005) in Pennsylvania. Of these, we found that first-generation cephalosporins (e.g., cephapirin) were the most commonly used, as was found by Pol and Ruegg (2007) in Wisconsin and by NAHMS in several states (USDA-APHIS, 2008). In contrast, Kuipers et al. (2016), Brunton et al. (2012), and Stevens et al. (2016) found that third-generation cephalosporins were most commonly used on dairy farms in Denmark, the United Kingdom, and Belgium, respectively. These differences could be due to country-specific prescribing patterns or management (e.g., use of blanket DCT versus targeted DCT; Biggs et al., 2016). We found that $85 \%$ of farms used DCT, with $61 \%$ reporting treating 
a)

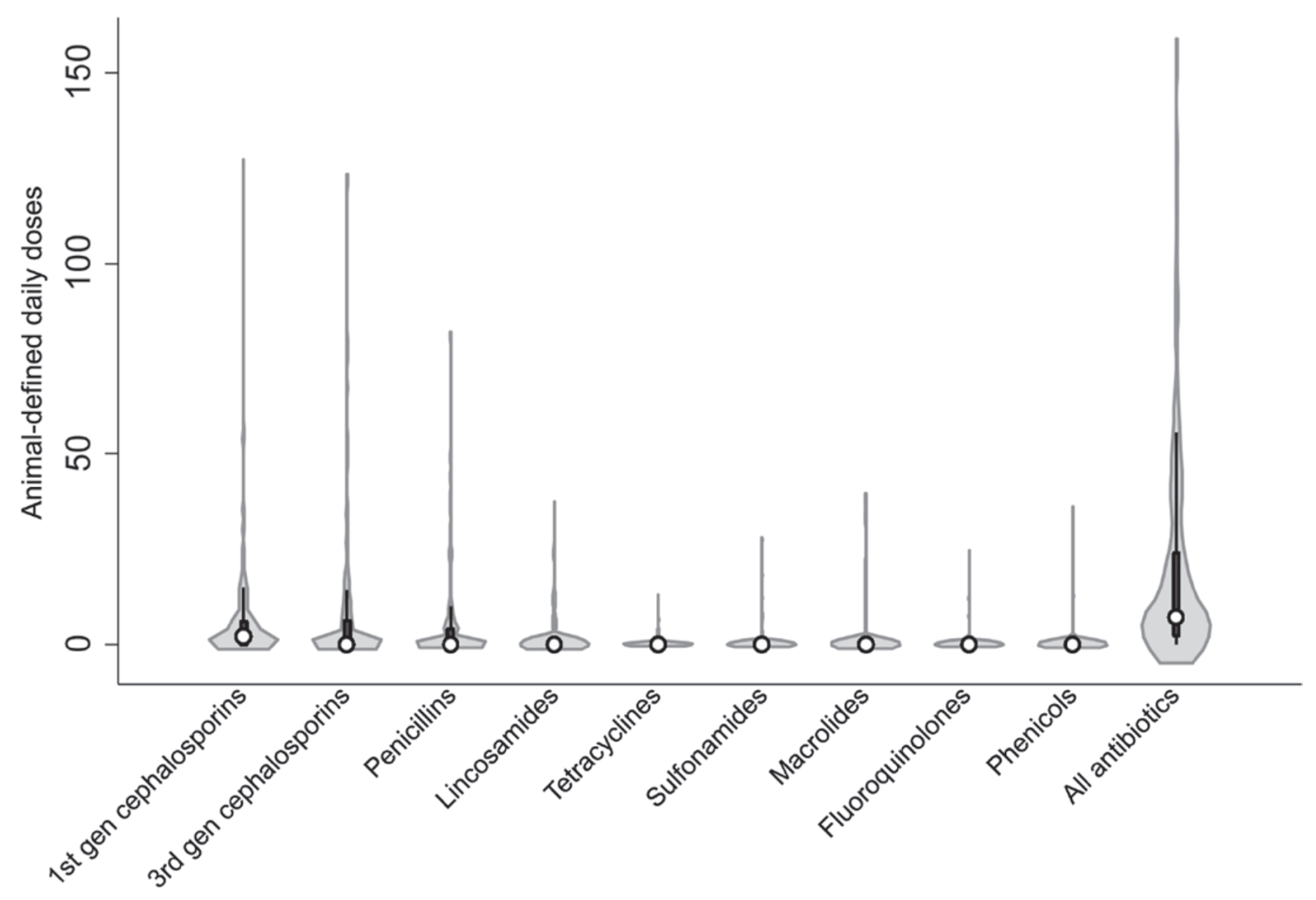

b)

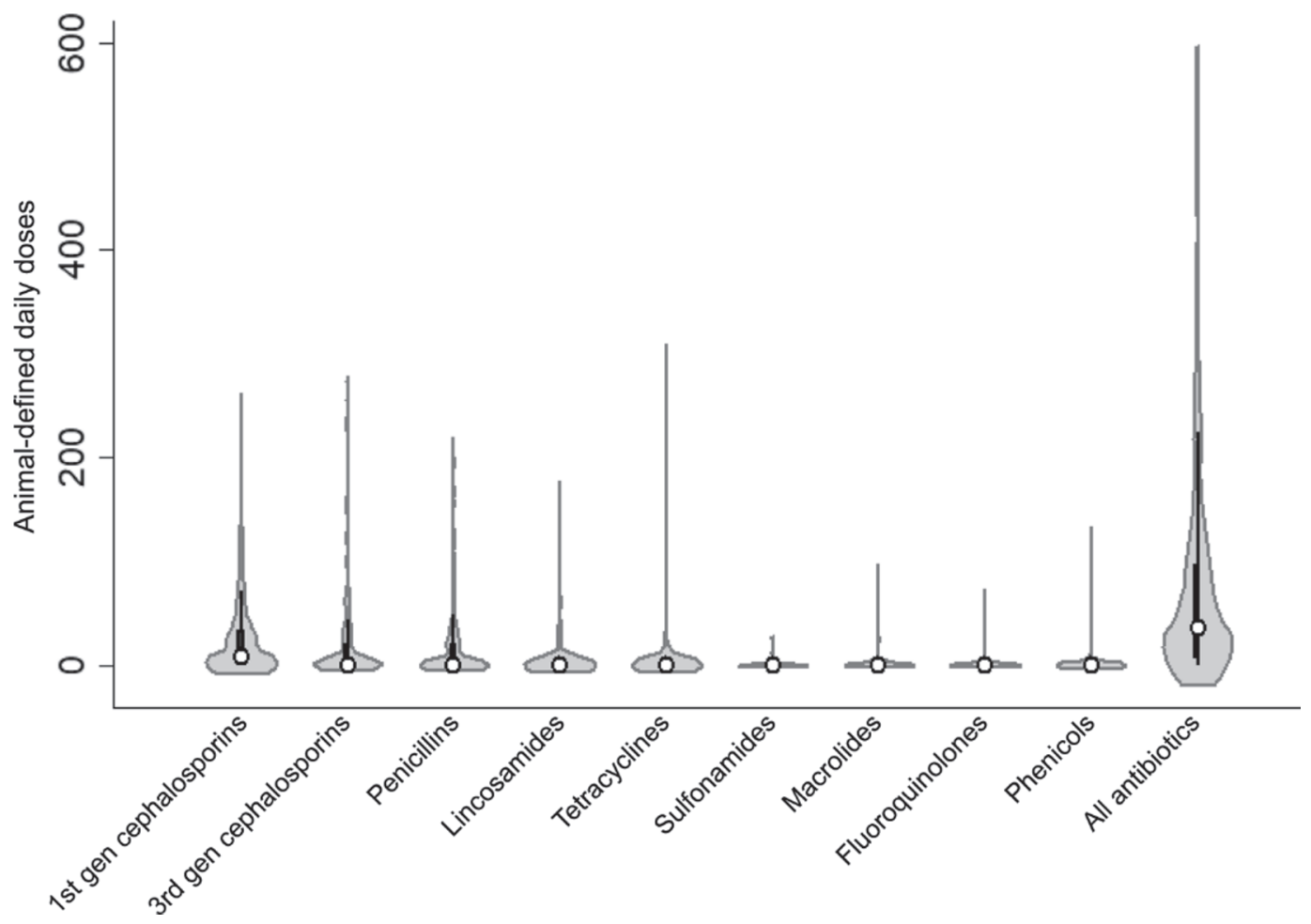

Figure 4. Violin plots of animal-defined daily doses by drug class used in the previous 1 mo (a) and 6 mo (b) on 235 dairy farms in Pennsylvania in 2017. The white circle represents the median, the vertical black bar represents the interquartile range, and the width of the body indicates the density. 
a)

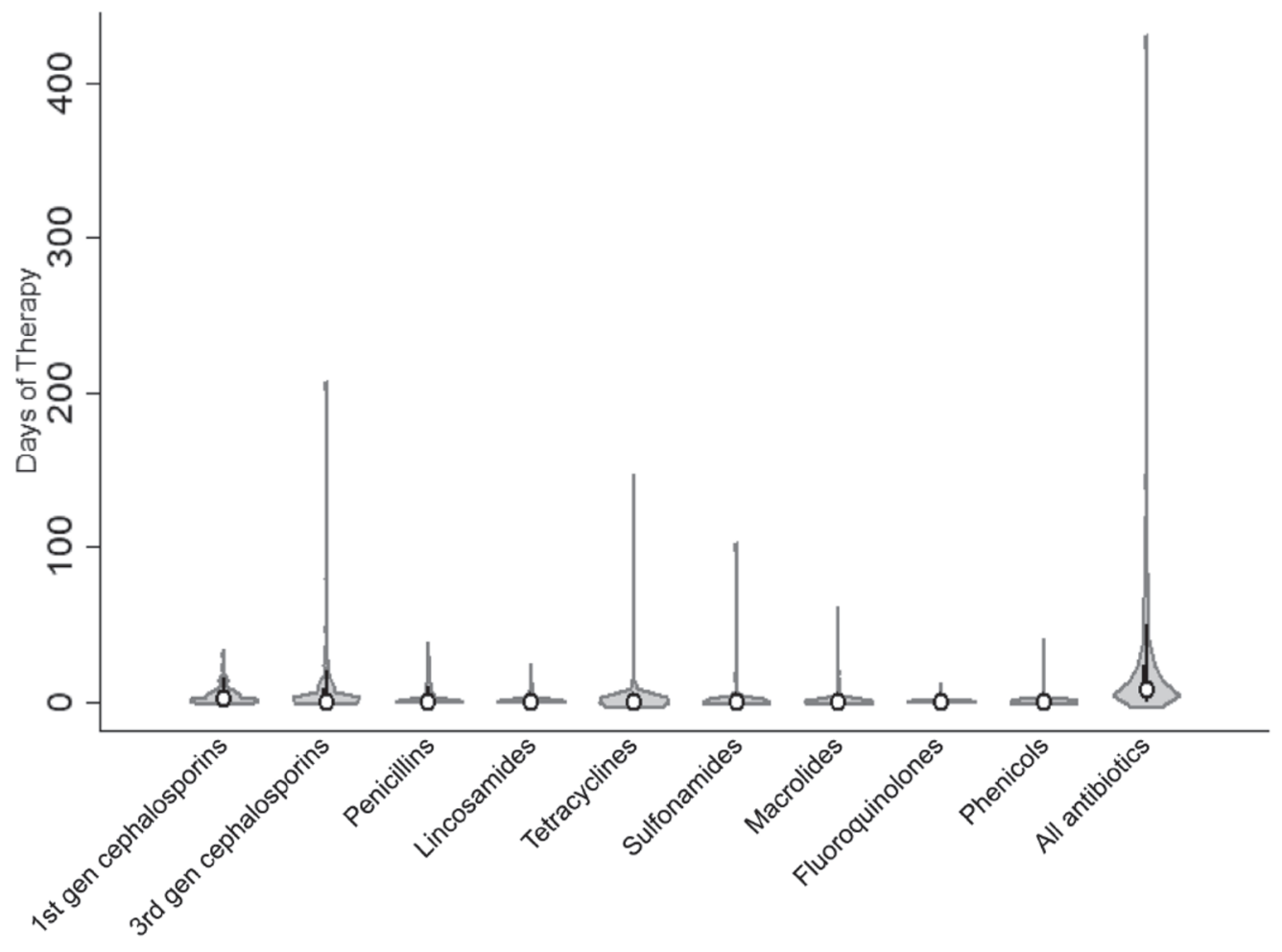

b)

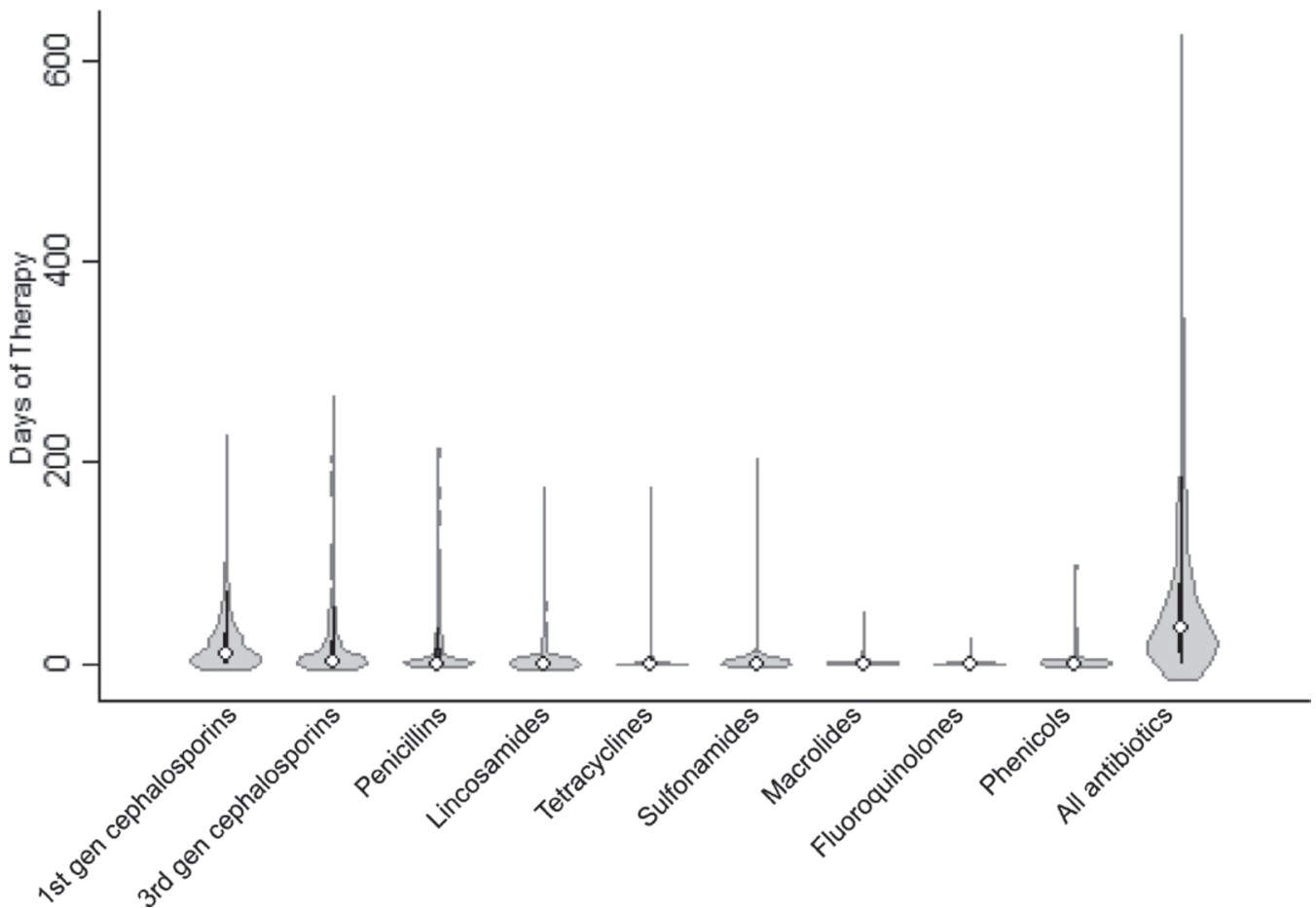

Figure 5. Violin plots of days of antibiotic therapy by drug class used in the previous 1 mo (a) and 6 mo (b) on 235 dairy farms in Pennsylvania in 2017. The white circle represents the median, the vertical black bar represents the interquartile range, and the width of the body indicates the density. 
a)

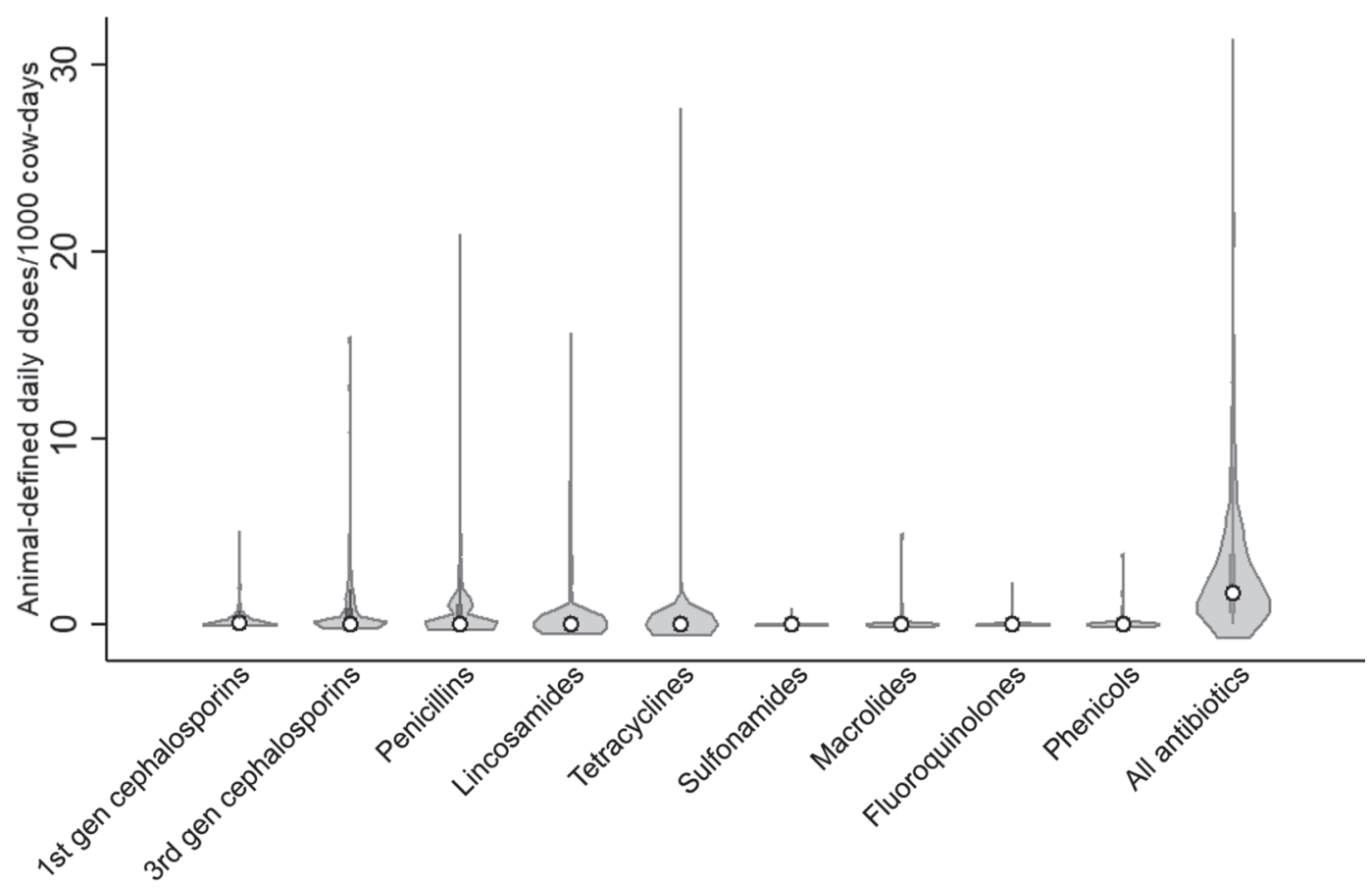

b)

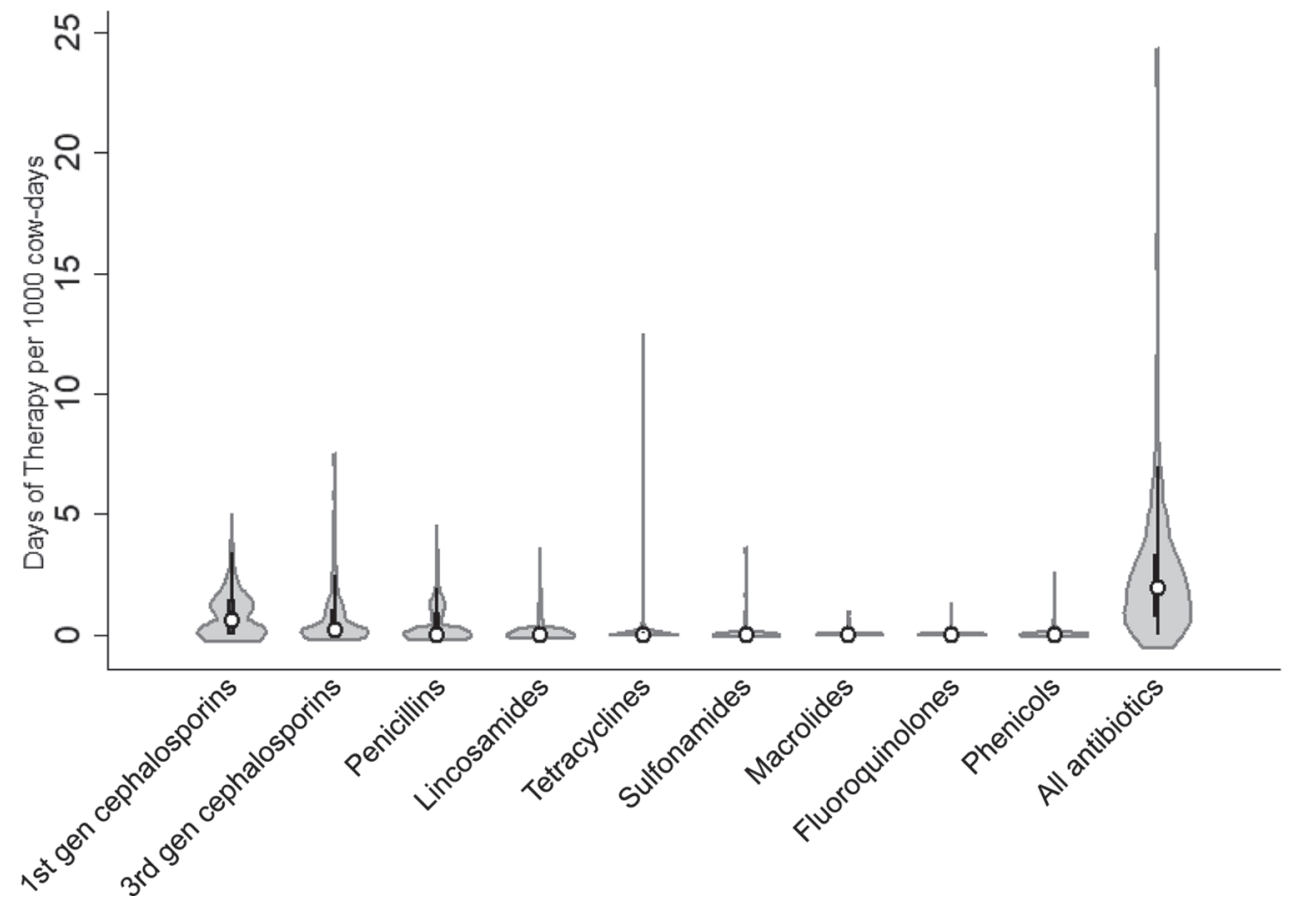

Figure 6. Violin plots of antibiotic treatment incidences by drug class used in the previous 6 mo on 235 dairy farms in Pennsylvania in 2017. (a) Animal-defined daily doses per 1,000 cow-days and (b) days of antibiotic use per 1,000 cow-days. The white circle represents the median, the vertical black bar represents the interquartile range, and the width of the body indicates the density. 
Table 5. Antibiotic treatment incidences ${ }^{1}$ per 1,000 animal-days by herd size on 235 dairy farms in Pennsylvania in 2017

\begin{tabular}{lccc}
\hline & & \multicolumn{2}{c}{$\begin{array}{c}\text { Median (range) treatment } \\
\text { incidence/1,000 animal-days }\end{array}$} \\
\cline { 3 - 4 } Herd size & Number & ADD & DOT \\
\hline Very small $(<30$ cows $)$ & $20(9)$ & $0(0-11)$ & $0.55(0-1.4)$ \\
Small (30-99 cows) & $162(70)$ & $1.7(0-67)$ & $1.9(0-18)$ \\
Medium (100-499 cows) & $50(21)$ & $2.3(0-55)$ & $2.7(0-25)$ \\
Large (500+ cows) & $2(1)$ & $3.8^{2}$ & 2.6 \\
\hline
\end{tabular}

${ }^{1} \mathrm{ADD}=$ animal defined daily dose; DOT $=$ days of therapy

${ }^{2}$ Doses and treatment durations were missing for the second of the large farms.

all of their cows (e.g., blanket DCT) and $15 \%$ treating 50 to $99 \%$ of their cows; NAHMS reported that 94 and $90 \%$ of farms used DCT in 2002 and 2007, respectively. In contrast, blanket DCT has been banned in several European countries, and is therefore likely used less frequently in the European countries where its use is still permitted.

The use of medicated milk replacers represents another nontherapeutic form of antibiotic use. Previously in the United States, medicated milk replacers were used extensively: the 2007 NAHMS report indicated that $57.5 \%$ of operations fed medicated milk replacers to their calves, with the most commonly used medication being a combination of oxytetracycline and neomycin (used on $49.5 \%$ of operations; USDA-APHIS, 2008). In contrast, we found that only $18.6 \%$ of farms used medicated milk replacer, and only 7 farms (3\%) used a milk replacer with antibiotics. This marked decrease may be explained by the fact that medicated milk replacers are now subject to the Veterinary Feed Directive and can no longer be obtained without a prescription.

As was found by Sawant et al. (2005) and Zwald et al. (2004), we did not find that the rate of treatment with antibiotics was associated with whether a farm kept treatment records. Previous studies have found that approximately $50 \%$ of farms keep treatment records (Ruegg, 2003; Zwald et al., 2004; USDA-APHIS, 2008). In this study, we found that $60 \%$ of farms maintained written records of antibiotic use and that herd size was not associated with the likelihood of having treatment records, suggesting that more farms are using written records to track antibiotic use.

As is the case with any study collecting antibiotic use data, this study has several limitations. First, this study relies on self-report. Studies quantifying antibiotic use on dairy farms have used different methods of collecting antibiotic use data, including veterinary prescription records (Kuipers et al., 2016; Obritzhauser et al., 2016), garbage can audits (Saini et al., 2012; Redding et al., 2014b), and producer recall/self-report (Zwald et al., 2004; Redding et al., 2014a). Recall of drug use data has been shown to produce under-reporting; West et al. (2006) compared self-reported use of nonsteroidal anti-inflammatory drugs (NSAID) in people over the previous $12 \mathrm{yr}$ with pharmacy dispensation records and found that the sensitivity of recall of any use of NSAID was $57 \%$. It can also be difficult to obtain specific quantitative (e.g., doses, volumes) and qualitative information (e.g., drug names or active ingredients) from recall. Recall is improved if the interview is performed closer to the index date, if the recall period encompasses a shorter period, and if the medication tends to be used relatively regularly (Lewis et al., 2006; Redding et al., 2014b). Kimmel et al. (2003) found that recall was enhanced by $6 \%$ when memory aids, such as having investigators read lists of medications and showing pictures of medications, were used. We used several of these techniques to enhance recall - the use of visual aids and having short recall periods (e.g., 1 and 6 mo). We used a recall period of 1 mo to enhance validity because we previously found that self-reported antibiotic drug use data for commonly used medications were highly correlated with garbage can audit data for a 1-mo recall period (Redding et al., 2014b). We used the longer recall period $(6 \mathrm{mo})$ to obtain a more representative snapshot of antibiotic use over the longer term and to improve generalizability of our findings to those of other studies, where collection periods tend to be $1 \mathrm{yr}$. Moreover, because $60 \%$ of surveyed producers reported maintaining treatment records, fewer farmers relied exclusively on recall. However, because our recall period was 6 mo and antibiotic use can be seasonal (Bishop et al., 1980; Laven and Lawrence, 2006; Moosavi et al., 2014; Kumar et al., 2016), our rates of antibiotic use may not accurately quantify the antibiotic use that occurs over the course of a year.

Another limitation is that we assumed the number of animals on the farm to be constant. Variation in the number of animals during different reporting periods can lead to an over- or underestimation of antibiotic consumption. Other authors have addressed this issue by using the mean number of animals at the begin- 
ning and end of a study period (González et al., 2010; Saini et al., 2012). However, because those authors also found that the total number of adult cattle varied little over the study period and because our study period was relatively short, we assumed that the number of dairy animals on the farm remained constant over the 6 -mo period.

Finally, we assumed that producers recorded or recalled all instances of antibiotic use. This might not be realistic if persons other than the producer were administering treatment (e.g., family members, workers, veterinarians). However, Sawant et al. (2005) reported that $93 \%$ of antibiotic treatments were given by farmers themselves in Pennsylvania. If this proportion has remained constant, then we can assume that self-report in combination with treatment records on $60 \%$ of farms captured a maximal number of treatments.

\section{CONCLUSIONS}

Rates of antibiotic use in the previous 1 and $6 \mathrm{mo}$ were relatively low on a sample of 235 dairy farms in Pennsylvania, and rates were significantly associated with farm size. These relatively low rates may have been related to the types of farms found in Pennsylvania (e.g., Amish and Mennonite farms, which tend to use antibiotics less frequently) or to the seasonality of antibiotic use. Poor recall of antibiotic use, especially by the $40 \%$ of farmers who did not maintain written treatment records, may have also contributed to the low rates, as could inaccuracies in calculated rates due to assumptions about animal weight and dosing levels. As reported in other studies, the main indication for antibiotic use was mastitis, and first-generation cephalosporins were the most commonly used class of antibiotic, followed by penicillins and third-generation cephalosporins. We noted a dramatic decrease in the number of farms feeding their calves medicated milk replacer. Different metrics were used to quantify antibiotic use, and these metrics were found to be highly correlated. We anticipate that our data can be used as a baseline to monitor temporal trends in antibiotic use on dairy farms in Pennsylvania and to evaluate the effect of interventions aimed at promoting the judicious use of antibiotics in the dairy industry.

\section{ACKNOWLEDGMENTS}

We thank the Center for Dairy Excellence for their assistance in providing contact information for the dairy producers. Funding for this study was provided by internal funds from the University of Pennsylvania School of Veterinary Medicine.

\section{REFERENCES}

Barlam, T. F., S. E. Cosgrove, L. M. Abbo, C. MacDougall, A. N Schuetz, E. J. Septimus, A. Srinivasan, T. H. Dellit, Y. T. FalckYtter, N. O. Fishman, C. W. Hamilton, T. C. Jenkins, P. A. Lipsett, P. N. Malani, L. S. May, G. J. Moran, M. M. Neuhauser, J. G. Newland, C. A. Ohl, M. H. Samore, S. K. Seo, and K. K. Trivedi. 2016. Implementing an antibiotic stewardship program: Guidelines by the Infectious Diseases Society of America and the Society for Healthcare Epidemiology of America. Clin. Infect. Dis. 62:e51-e77.

Biggs, A., D. Barrett, A. Bradley, M. Green, K. Reyher, and R. Zadoks. 2016. Antibiotic dry cow therapy: Where next? Vet. Rec. 178:93-94.

Bishop, J. R., A. B. Bodine, and J. J. Janzen. 1980. Sensitivities to antibiotics and seasonal occurrence of mastitis pathogens. J. Dairy Sci. 63:1134-1137.

Brunton, L. A., D. Duncan, N. G. Coldham, L. C. Snow, and J. R. Jones. 2012. A survey of antimicrobial usage on dairy farms and waste milk feeding practices in England and Wales. Vet. Rec. 171:296.

Bryan, M., and S. Y. Hea. 2017. A survey of antimicrobial use in dairy cows from farms in four regions of New Zealand. N. Z. Vet. J. 65:93-98.

Center for Dairy Excellence. 2018. Dairy information. Center for Dairy Excellence, Harrisburg, PA. Accessed Jul. 1, 2018. http:// centerfordairyexcellence.org/dairy-information.

European Medicines Agency. 2015. Principles on assignment of defined daily dose for animals (DDDA) and defined course dose for animals (DCDA). Accessed Jul 1, 2018. http://www.ema.europa .eu/docs/en_GB/document_library/Scientific_guideline/2015 03/WC500184369.pdf.

Food and Drug Administration (FDA). 2009. Guidance for Industry-Veterinary Feed Directive Regulation Questions and Answers Final Guidance. 2009. Accessed Nov. 28, 2018. https:// www.federalregister.gov/documents/2015/06/03/2015-13393/ veterinary-feed-directive.

González, S. M., A. Steiner, B. Gassner, and G. Regula. 2010. Antimicrobial use in Swiss dairy farms: Quantification and evaluation of data quality. Prev. Vet. Med. 95:50-63.

Harris, P. A., R. Taylor, R. Thielke, J. Payne, N. Gonzalez, and J. G. Conde. 2009. Research electronic data capture (REDCap)-A metadata-driven methodology and workflow process for providing translational research informatics support. J. Biomed. Inform. $42: 377-381$

Hyde, R. M., J. G. Remnant, A. J. Bradley, J. E. Breen, C. D. Hudson, P. L. Davies, T. Clarke, Y. Critchell, M. Hylands, E. Linton, E. Wood, and M. J. Green. 2017. Quantitative analysis of antimicrobial use on British dairy farms. Vet. Rec. 181:683.

Jensen, V. F., E. Jacobsen, and F. Bager. 2004. Veterinary antimicrobial-usage statistics based on standardized measures of dosage. Prev. Vet. Med. 64:201-215.

Kimmel, S. E., J. D. Lewis, J. Jaskowiak, L. Kishel, and S. Hennessy. 2003. Enhancement of medication recall using medication pictures and lists in telephone interviews. Pharmacoepidemiol. Drug Saf. $12: 1-8$.

Kuipers, A., W. J. Koops, and H. Wemmenhove. 2016. Antibiotic use in dairy herds in the Netherlands from 2005 to 2012. J. Dairy Sci. 99:1632-1648

Kumar, N., A. Manimaran, A. Kumaresan, L. Sreela, T. K. Patbandha, S. Tiwari, and S. Chandra. 2016. Episodes of clinical mastitis and its relationship with duration of treatment and seasonality in crossbred cows maintained in organized dairy farm. Vet. World 9:75-79.

Laven, R. A., and K. R. Lawrence. 2006. An evaluation of the seasonality of veterinary treatments for lameness in UK dairy cattle. J. Dairy Sci. 89:3858-3865.

Léger, D. F., N. C. Newby, R. Reid-Smith, N. Anderson, D. L. Pearl, K. D. Lissemore, and D. F. Kelton. 2017. Estimated antimicrobial dispensing frequency and preferences for lactating cow therapy by Ontario dairy veterinarians. Can. Vet. J. 58:26-34. 
Lewis, J. D., B. L. Strom, S. E. Kimmel, J. Farrar, D. C. Metz, C. Brensinger, L. Nessel, and A. R. Localio. 2006. Predictors of recall of over-the-counter and prescription non-steroidal anti-inflammatory drug exposure. Pharmacoepidemiol. Drug Saf. 15:39-45.

Mills, H. L., A. Turner, L. Morgans, J. Massey, H. Schubert, G. Rees, D. Barrett, A. Dowsey, and K. K. Reyher. 2018. Evaluation of metrics for benchmarking antimicrobial use in the UK dairy industry. Vet. Rec. 182:379.

Moosavi, M., A. Mirzaei, M. Ghavami, and A. Tamadon. 2014. Relationship between season, lactation number and incidence of clinical mastitis in different stages of lactation in a Holstein dairy farm. Vet. Res. Forum 5:13-19.

Obritzhauser, W., M. Trauffler, J. Raith, I. Kopacka, K. Fuchs, and J. Kofer. 2016. Antimicrobial drug use on Austrian dairy farms with special consideration of the use of "highest priority critically important antimicrobials". Berl. Munch. Tierarztl. Wochenschr. 129:185-195

Pol, M., and P. L. Ruegg. 2007. Treatment practices and quantification of antimicrobial drug usage in conventional and organic dairy farms in Wisconsin. J. Dairy Sci. 90:249-261.

Raymond, M. J., R. D. Wohrle, and D. R. Call. 2006. Assessment and promotion of judicious antibiotic use on dairy farms in Washington State. J. Dairy Sci. 89:3228-3240.

Redding, L. E., F. Cubas-Delgado, M. D. Sammel, G. Smith, D. T. Galligan, M. Z. Levy, and S. Hennessy. 2014a. The use of antibiotics on small dairy farms in rural Peru. Prev. Vet. Med. 113:88.

Redding, L. E., F. Cubas-Delgado, M. D. Sammel, G. Smith, D. T. Galligan, M. Z. Levy, and S. Hennessy. 2014b. Comparison of two methods for collecting antibiotic use data on small dairy farms. Prev. Vet. Med. 114:213-222.

Ruegg, P. L. 2003. Investigation of mastitis problems on farms. Vet. Clin. North Am. Food Anim. Pract. 19:47-73.

Saini, V., J. T. McClure, D. Leger, S. Dufour, A. G. Sheldon, D. T. Scholl, and H. W. Barkema. 2012. Antimicrobial use on Canadian dairy farms. J. Dairy Sci. 95:1209-1221.
Sawant, A. A., L. M. Sordillo, and B. M. Jayarao. 2005. A survey on antibiotic usage in dairy herds in Pennsylvania. J. Dairy Sci. 88:2991-2999.

Schewe, R. L., and C. Brock. 2018. Stewarding dairy herd health and antibiotic use on U.S. Amish and Plain Mennonite farms. J. Rural Stud. 58:1-11.

Stevens, M., S. Piepers, K. Supre, J. Dewulf, and S. De Vliegher. 2016. Quantification of antimicrobial consumption in adult cattle on dairy herds in Flanders, Belgium, and associations with udder health, milk quality, and production performance. J. Dairy Sci. 99:2118-2130.

USDA-APHIS. 2008. Antibiotic use on US Dairy Operations, 2002 and 2007. Accessed May 5, 2018. http://nahms.aphis.usda.gov/dairy/ index.htm\#dairy2007.

USDA-APHIS-NAHMS. 2016. Dairy Cattle Management Practices in the United States, 2014. Accessed May 5, 2018. https://www .aphis.usda.gov/animal_health/nahms/dairy/downloads/dairy14/ Dairy14_dr_PartI.pdf.

West, S., B. Strom, and C. Poole. 2006. Validity of pharmacoepidemiologic drug and diagnosis data. Pages 239-258 in Textbook of Pharmacoepidemiology. B. Strom and S. Kimmel, ed. Wiley, Chichester, UK.

WHO (World Health Organization). 2013a. Critically important antimicrobials for human medicine. WHO, Geneva, Switzerland. http: //www.who.int/foodsafety/areas_work/antimicrobial-resistance/ cia/en/.

WHO (World Health Organization). 2013b. Guidelines for ATC classification and DDD assignment. https://www.whocc.no/filearchive/ publications/guidelines.pdf.

Zwald, A. G., P. L. Ruegg, J. B. Kaneene, L. D. Warnick, S. J. Wells, C. Fossler, and L. W. Halbert. 2004. Management practices and reported antimicrobial usage on conventional and organic dairy farms. J. Dairy Sci. 87:191-201. 